SNUTP 94-02

\title{
Small Flavor Conserving CP Violation in Superstring Models*
}

\author{
Kiwoon Choi \\ Department of Physics, Chonbug National University \\ Chonju 560-756, KOREA
}

\begin{abstract}
It is well known that supersymmetric models allow new sources for CP violation that arise from soft supersymmetry breaking terms. If unsuppressed, these new CP-violating phases would give too large a neutron electric dipole moment. We discuss a mechanism for suppressing SUSY phases in stringinspired supergravity models in which supersymmetry is assumed to be broken by the auxiliary components of the dilaton and moduli superfields.
\end{abstract}

\section{INTRODUCTION}

Since the first experimental observation of CP violation, the flavor (strangeness) changing decays of $K$ mesons remain to be the only place in which the violation has been seen experimentally. Although not observed yet, flavor conserving $\mathrm{CP}$ violation, e.g. particle electric dipole moments, is also of great interest since it can provide useful information on physics beyond the standard model.

As is well known, the standard model contains only two sources for $\mathrm{CP}$ violation, the Kobayashi-Maskawa $(\mathrm{KM})$ phase $\delta_{\mathrm{KM}}$ and the QCD vacuum angle $\bar{\theta}$. To explain the ob-

\footnotetext{
*To appear in the Proceeding of the Third KEK Topical Conference on CP Violation, its Implication to Particle Physics and Cosmology
} 
served CP violation in $K$-decays, $\delta_{\mathrm{KM}}$ is required to be of order unity. Even for $\delta_{\mathrm{KM}}$ of order unity, the resulting flavor conserving CP violation is negligibly small because it is second order in weak interactions while its CP conserving counterpart occurs at zeroth order without resorting to weak interactions. Note that although $\triangle S=2 \mathrm{CP}$-odd $K-\bar{K}$ mixing is second order in weak interactions, its CP-even counterpart is second order also, allowing the experimental observation of $\epsilon \sim$ odd/even $\sim 10^{-3}$ where the small value of $\epsilon$ is due to small mixing angles, not due to a small $\delta_{\mathrm{KM}}$.

Contrary to this, to be compatible with the current experimental limit on the neutron electric dipole moment $d_{n} \leq 10^{-25} e \cdot \mathrm{cm}, \bar{\theta}$ is required to be less than $10^{-9}$ [1]. For $\bar{\theta}=$ $\theta_{\mathrm{QCD}}+\theta_{\mathrm{QFD}}$, both $\theta_{\mathrm{QFD}}=\arg \left(\operatorname{det} M_{q}\right)$ and $\delta_{\mathrm{KM}}$ originate from complex Yukawa couplings, and thus are expected to have the same order of magnitudes. Then the phenomenological limit on $\bar{\theta}$ requires fine tuning $\theta_{\mathrm{QCD}}$ to cancel $\theta_{\mathrm{QFD}}$. The strong $\mathrm{CP}$ problem of this fine tuning has motivated a variety of extensions to the standard model which accommodate a mechanism for setting $\bar{\theta}$ to zero [1].

In recent years, supersymmetry (SUSY) has emerged as a leading candidate for physics beyond the standard model around the weak scale. This is largely due to the fact that SUSY provides a perturbative solution to the problem of the quadratic divergence in scalar masses. Furthermore if SUSY breaking is triggered by nonperturbative effects, supersymmetry may also provide an explanation for the large hierarchy between the weak scale and the Planck scale [2].

Although quite attractive in view of the hierarchy problem, conventional supersymmetric models suffer from several naturalness problem not shared by the standard model. For instance, they require a high level of degeneracy in squark and slepton masses to avoid too large flavor changing neutral currents. This is hard to be understood in view of a rather large hierarchy in quark and lepton masses. Besides this SUSY flavor problem, there is another naturalness problem, the "SUSY phase problem" [3], which is the subject of this talk. As is well known, supersymmetric models allow new sources for CP violation arising from soft SUSY breaking terms. For superpartner masses around the weak scale, these new 
SUSY phases are required to be less than $10^{-2}-10^{-3}$ in order to avoid too large a neutron electric dipole moment [3]. Although not as severe as $\bar{\theta}$, it is still hard to understand how theories can give rise to small SUSY phases while giving the KM phase of order unity.

Clearly the SUSY phase problem is a problem of SUSY breaking since the relevant phases originate from soft SUSY breaking terms. Presently the most popular way to break SUSY is to introduce a hidden sector into the underlying $N=1$ supergravity model 《. Four dimensional $N=1$ supergravity theories are highly nonrenormalizable and thus are considered to be an effective theory of a more fundamental theory which is presumed to consistently unify the gravity with particle physics interactions. Presently string theory is the only known candidate along this direction [5]. In this regard, it would be quite interesting to explore the possibility of small SUSY phases in string-inspired supergravity models.

Here we wish to discuss a mechanism for suppressing SUSY phases which would be realized in string-inspired models if some plausible conditions are met [6]. The essential ingredients of the mechanism are: (i) approximate Peccei Quinn symmetries nonlinearly realized for the pseudoscalar components of the moduli superfields which contribute to SUSY breaking by having nonzero auxiliary components, (ii) dynamical relaxation of the relative

phases in the nonperturbative superpotential of the dilaton and moduli superfields. The conditions for the mechanism to work are not so restrictive, and are satisfied in fact by many SUSY breaking scenarios in string theories [7, [1].

\section{THE SMALL SUSY PHASE PROBLEM}

To begin with, let us consider a generic renormalizable supersymmetric model with the effective superpotential

$$
W_{\mathrm{eff}}=\lambda_{i j k} \Phi_{i} \Phi_{j} \Phi_{k}+\mu_{i j} \Phi_{i} \Phi_{j},
$$

and the soft breaking terms 


$$
\begin{aligned}
\mathcal{L}_{\text {soft }}= & \frac{1}{2} m_{i j}^{2} \varphi_{i} \varphi_{j}^{*}+A_{i j k} \varphi_{i} \varphi_{j} \varphi_{k} \\
& +B_{i j} \varphi_{i} \varphi_{j}+\frac{1}{2} m_{a} \lambda_{a} \lambda_{a}+\text { h.c. },
\end{aligned}
$$

where $\Phi_{i}$ denote generic chiral superfields, $\varphi_{i}$ are their scalar components, and $\lambda_{a}$ are the gaugino for the $a$-th gauge group.

All the parameters that appear in $W_{\text {eff }}$ and $\mathcal{L}_{\text {soft }}$ are complex in general, and thus can give rise to $\mathrm{CP}$-violating phenomena in principle. However physical $\mathrm{CP}$ violation occurs only through CP-odd parameters which can not be eliminated by reparametrizing field variables. The hermitian mass matrix $m_{i j}^{2}$ can always be made to be real and diagonal by a unitary transformation of superfields: $\Phi_{i} \rightarrow U_{i j} \Phi_{j}$. One can still perform an arbitrary phase rotation of superfields, $U(1)_{i}: \Phi_{i} \rightarrow e^{i \alpha_{i}} \Phi_{i}$, to eliminate some of the phases while keeping $m_{i j}^{2}$ real and diagonal. Clearly the following three classes of phases

$$
\begin{aligned}
& \phi_{A}=\left\{\arg \left(A_{i j k} / \lambda_{i j k}\right)\right\}, \\
& \phi_{B}=\left\{\arg \left(B_{i j} / \mu_{i j}\right)\right\}, \\
& \phi_{C}=\left\{\arg \left(m_{a}\right)\right\}
\end{aligned}
$$

are invariant under $U(1)_{i}$, and thus are the candidates for the sources for physical CP violation. There is a further transformation which allows one of the above phases to be rotated away. It is an $R$-transformation $U(1)_{R}$ :

$$
\Theta \rightarrow e^{i \alpha} \Theta, \quad \Phi_{i} \rightarrow \Phi_{i}
$$

where $\Theta$ denotes the Grassmann coordinate of superspace. Obviously $\phi_{A, B, C} \rightarrow \phi_{A, B, C}+2 \alpha$ under $U(1)_{R}$, implying that one of the phases can be eliminated by an appropriate $U(1)_{R}$ transformation.

The above discussion shows that a number of $\mathrm{CP}$ violating phases can arise from soft supersymmetry breaking terms. Inspired by simple hidden sector supergravity models, the following universality conditions are often assumed for the coefficients of soft terms renormalized at the Planck scale: 


$$
A_{i j k}=A \lambda_{i j k}, \quad B_{i j}=B \mu_{i j}, \quad m_{a}=\tilde{m} .
$$

In this simple case, soft supersymmetry breaking terms provide only two new reparametrization-invariant $\mathrm{CP}$-violating phases which can be chosen to be [9]

$$
\begin{aligned}
\phi & =\left\{\arg \left(\tilde{m} A^{*}\right), \arg \left(\tilde{m} B^{*}\right)\right\} \\
& =\left\{\phi_{C}-\phi_{A}, \phi_{C}-\phi_{B}\right\}
\end{aligned}
$$

Supersymmetric lagrangian contains a squark-quark-gluino coupling $I_{q \tilde{q} \lambda} \sim \tilde{q}^{*} T^{a} q \lambda^{a}$ which is manifestly CP-conserving when written in terms of weak eigenstate fields. Due to soft SUSY breaking terms, the unitary matrix relating the weak eigenstate quarks to the mass eigenstate quarks is different from the unitary matrix for squarks. As a result, the coupling $I_{q \tilde{q} \lambda}$ written in terms of mass eigenstate fields would contain flavor-conserving but CP-violating piece which depends upon the phase $\phi$. Radiative effects of this coupling then induce flavor-conserving but CP-violating effective operators of quarks and gluons. In regard to the neutron electric dipole moment $d_{n}$, the operators of interest are the electric dipole moments (EDM) of light quarks and the chromo-electric dipole moments (CEDM) of light quarks and gluon:

$$
\begin{aligned}
& \mathcal{O}_{q}^{E}=d_{q}^{E} \frac{m_{q}}{M_{W}^{2}} e F_{\mu \nu} \bar{q} i \sigma^{\mu \nu} \gamma_{5} q, \\
& \mathcal{O}_{q}^{C}=d_{q}^{C} \frac{m_{q}}{M_{W}^{2}} g_{c} G_{\mu \nu}^{a} \bar{q} i \sigma^{\mu \nu} \gamma_{5} T^{a} q, \\
& \mathcal{O}_{g}^{C}=d_{g}^{C} \frac{1}{M_{W}^{2}} g_{c}^{3} f_{a b c} G_{\mu \alpha}^{a} G_{\nu}^{b \alpha} G_{\rho \sigma}^{c} \epsilon^{\mu \nu \rho \sigma} .
\end{aligned}
$$

The EDM and CEDM of $q$ are induced by one-loop graphs involving the internal squark $\tilde{q}$ and the gluino $\lambda^{a}$ attached to the external $q$ via the coupling $I_{q \tilde{q} \lambda}$, while the gluon CEDM is due to two loop graphs involving the internal $t, \tilde{t}$ and $\lambda^{a}$. Note that the EDM and CEDM of $q$ change the chirality of $q$, and thus are suppressed by the small quark mass $m_{q}$. The EDM of light quarks in supersymmetric models was computed a decade ago [10] while the potential importance of the gluon CEDM was noted rather recently by Weinberg [11]. 
Without including perturbative QCD corrections, one can naively estimate the contributions to $d_{n}$ from the above three operators. Since $\mathcal{O}_{g}^{C}$ is a two-loop result while $\mathcal{O}_{q}^{E, C}$ are one-loop results, one roughly expects $d_{q}^{E} \sim d_{q}^{C} \sim 16 \pi^{2} d_{g}^{C}$, implying

$$
d_{n}\left(\mathcal{O}_{q}^{E}\right) \sim d_{n}\left(\mathcal{O}_{q}^{C}\right) \sim \xi d_{n}\left(\mathcal{O}_{g}^{C}\right)
$$

where $\xi=\left(m_{q} / M_{n}\right)\left(4 \pi / \alpha_{c}\right)$ for the neutron mass $M_{n}$. Since $\xi$ is roughly of order unity, one needs a more detailed analysis including QCD corrections in order to see which of the operators gives a dominant contribution. Recently the neutron EDM resulting from the operators of eq. (7) in supersymmetric models was reassessed in ref. [12]. The result indicates that the contribution from $\mathcal{O}_{q}^{E}$ slightly dominates other contributions, yielding

$$
d_{n}\left(\mathcal{O}_{q}^{E}\right) \simeq 10^{-22} \eta_{q}\left(\frac{100 \mathrm{GeV}}{M_{S}}\right)^{2} \sin \phi e \cdot c m
$$

where $M_{S}$ denotes the superpartner masses which are assumed to have a common value, and $\eta_{q}=c_{q} Q_{q}^{\mathrm{em}} m_{q} / 6 \mathrm{MeV}$ for the electromagnetic charge $Q_{q}^{\mathrm{em}}$ of $q$ and the matrix element $c_{q}$ which is defined by $\left\langle n\left|\bar{q} \sigma_{\mu \nu} q\right| n\right\rangle=c_{q} \bar{n} \sigma_{\mu \nu} n$. One then has

$$
\phi \leq 10^{-3} \eta_{q}^{-1}\left(\frac{M_{S}}{100 \mathrm{GeV}}\right)^{2}
$$

for the current experimental limit of $d_{n} \leq 10^{-25} e \cdot \mathrm{cm}$.

For the above constraint to be satisfied, one needs either $\phi$ to be small enough or $M_{S}$ to be significantly larger than $100 \mathrm{GeV}$. For instance, for $M_{S}=100 \mathrm{GeV}$ and $q=u$ for which $\eta_{u} \simeq 1$, one has the limit $\phi \leq 10^{-3}$. If there exists a significant amount of the sea strange quarks in the neutron and thus $c_{s} m_{s}$ is significantly greater than $c_{u} m_{u}$, whose possibility has been argued in the literatures [13], one would obtain an even stronger limit [14]. Clearly the bound on $\phi$ can be relaxed by assuming that the superpartners have masses larger than $100 \mathrm{GeV}$. For instance, if $M_{S}$ is about a few $\mathrm{TeV}, \phi$ can be of order unity. Although the option of heavy superpartners is still allowed [15], a phenomenologically more desirable possibility is to have $\phi \leq 10^{-2}-10^{-3}$ while the superpartner masses are remained to be around a few hundreds $\mathrm{GeV}$ [3]. It is then hard to understand how theories can give rise to 
small SUSY phases while giving the KM phase of order unity. The required smallness of $\phi$ is less severe than that of $\bar{\theta}$, but this problem of small SUSY phase persists even in models with a mechanism, e.g. axions, for setting $\bar{\theta}$ to zero.

\section{SOME FEATURES OF STRING-INSPIRED SUPERGRAVITY}

Let us briefly discuss some features of string-inspired supergravity models which will be relevant for our later discussion of small SUSY phases. The model we consider here has the following properties which are believed to be generic properties of superstring vacua. First of all, the model contains a hidden sector which provides nonperturbative dynamics for SUSY breaking. This nonperturbative hidden sector generally has a large gauge group as well as matter fields that transform nontrivially under the hidden sector gauge group.

The model contains also the dilaton superfield $S$ and the overall modulus superfield $T$ [5]. The dilaton scalar component $\operatorname{Re}(S)$ couples to the gauge kinetic terms and thus determines the gauge coupling constant $g^{2}=1 / \operatorname{Re}(S)$. The overall modulus $\operatorname{Re}(T)$ corresponds to the radius of the compactified internal space in the Planck length unit. Then $1 / \operatorname{Re}(T)$ would determine the coupling constant of the world sheet sigma model for strings propagating in the internal space. In fact, $S$ and $T$ can be considered as members of hidden sector in the sense that they couple to observable sector by a gravitational strength. However here we distinguish them from the other type of hidden sector providing nonperturbative dynamics for SUSY breaking.

Compactified superstring predicts moduli fields other than $T$ in general. In this section, we ignore such moduli for the sake of simplicity. The effects of including other moduli will be discussed later. Then the key assumption of the model is that local SUSY is broken by the auxiliary $F$-components of $S$ and/or $T$, which are induced essentially by the nonperturbative hidden sector which couples to $S$ and $T$ by a gravitational strength.

A property of $S$ and $T$ which is crucial for our later discussion is that in perturbation theory their pseudoscalar components, the model-independent axion $\operatorname{Im}(S)$ and the internal 
axion $\operatorname{Im}(T)$, decouple at zero four momentum [5]. In spacetime perturbation theory in which the background gauge field configuration is topologically trivial, the vertex operators of these axion-like fields at zero four momentum are given by:

$$
\begin{aligned}
V_{\operatorname{Im}(S)} & =\int d^{2} \sigma \epsilon^{\alpha \beta} B_{\mu \nu} \partial_{\alpha} X^{\mu} \partial_{\beta} X^{\nu}, \\
V_{\operatorname{Im}(T)} & =\int d^{2} \sigma \epsilon^{\alpha \beta} B_{m n} \partial_{\alpha} Y^{m} \partial_{\beta} Y^{n},
\end{aligned}
$$

where $X^{\mu}$ and $Y^{m}$ denote the string coordinates of the flat Minkowski spacetime and the internal space respectively, $B_{\mu \nu}$ is a constant two-form, and $B_{m n}$ is the Kähler form of the internal space. Clearly $V_{\operatorname{Im}(S)}$ is a total divergence and thus vanishes. However $V_{\operatorname{Im}(T)}$ vanishes only for $Y^{m}$ which corresponds to a topologically trivial mapping from the string world sheet to the internal space. This implies that the constant mode of $\operatorname{Im}(S)$ decouples in spacetime perturbation theory ignoring Yang-Mills instanton fluctuations, while that of $\operatorname{Im}(T)$ decouples in a more restrictive case ignoring both Yang-Mills and world sheet instanton fluctuations [16].

The decoupling of the constant modes of $\operatorname{Im}(S)$ and $\operatorname{Im}(T)$ means the invariance under the nonlinear Peccei Quinn (PQ) symmetries:

$$
\begin{aligned}
& U(1)_{S}: S \rightarrow S+i \alpha_{S}, \\
& U(1)_{T}: T \rightarrow T+i \alpha_{T},
\end{aligned}
$$

where $\alpha_{S, T}$ are arbitrary real constants. In the early stage of anomaly free superstring theories, it has been speculated that the model-independent axion $\operatorname{Im}(S)$ and/or the internal axion $\operatorname{Im}(T)$ may solve the strong CP problem [17], barring the cosmological difficulty associated with too large decay constants [18]. However to be useful for the strong CP problem, explicit breaking of $U(1)_{S}$ or of $U(1)_{T}$ must be highly dominated (by a factor larger than $10^{9}$ ) by the gluon anomaly $G \tilde{G}$. For $U(1)_{S}$, this would be achieved in an uninteresting case that the color $S U(3)$ is the only confining non-abelian gauge group of the model. However in cases with non-abelian hidden sector gauge group, PQ symmetries are broken either by hidden sector Yang-Mills instantons or by world sheet instantons, whose effects 
are much stronger than that of the gluon anomaly in general. Since non-abelian hidden sector gauge group is strongly motivated for SUSY breaking, the axion-like fields $\operatorname{Im}(S)$ and $\operatorname{Im}(T)$ are considered to be irrelevant for the strong CP problem. However as we will see in the next section they can be very useful for solving another naturalness problem, the small SUSY phase problem.

We already noted that $U(1)_{S}$ is broken by Yang-Mills instantons including those of the hidden sector gauge group, and $U(1)_{T}$ is by both Yang-Mills and world sheet instantons. Since $1 / \operatorname{Re}(S)$ and $1 / \operatorname{Re}(T)$ correspond to the gauge coupling constant and the sigma model coupling constant respectively, the effects of Yang-Mills instantons would be suppressed by $q_{S} \equiv e^{-b_{S} S}$ while those of world sheet instantons are by $q_{T} \equiv e^{-b_{T} T}$ where $b_{S}$ and $b_{T}$ are some real constants. For $S$ normalized as $1 / \operatorname{Re}(S)=g^{2}$ (at string tree level), one has $b_{S}$ of order $4 \pi^{2}$, leading to $q_{S} \ll 10^{-3}$ for phenomenologically desirable $g^{2} \simeq 1$. A common normalization of $T$ is $T \equiv T+i$ for which $b_{T}=2 \pi$. In the following, we will assume that $q_{T}=e^{-2 \pi T} \leq 10^{-2}-10^{-3}$ or even less, so that for the estimate of SUSY phases up to the accuracy of $10^{-2}-10^{-3}$, PQ-violating effects can be ignored if they do not correspond to leading effects, but just give small corrections to perturbative PQ-conserving parts. Of course to justify this assumption, one needs to determine the vacuum value of the modulus $\operatorname{Re}(T)$ by evaluating the effective potential. This has been done for some orbifold models, yielding $\operatorname{Re}(T) \simeq 1.2$ 8, 19] for which $q_{T} \leq 10^{-3}$. At any rate, it is quite conceivable that $q_{T} \leq 10^{-2}-10^{-3}$ since $q_{T}$ is exponentially small for a moderately large value of $\operatorname{Re}(T)$.

As is well known, a four-dimensional $N=1$ supergravity action is characterized by the Kähler potential $K$, the superpotential $W$, and the gauge kinetic function $f_{a}$ for the $a$-th gauge group. Let us start with a generic supergravity model containing nonperturbative hidden sector which provides a seed for supersymmetry breaking. By our assumption, the nonperturbative hidden sector of the model does not directly develop SUSY breaking vacuum values. Then the effective theory obtained by integrating out the nonperturbative hidden sector would still have the structure of $N=1$ supergravity. The Kähler potential and the superpotential of this effective supergravity can be expanded in observable chiral superfields 
$\Phi_{i}$ as

$$
\begin{aligned}
& K=\tilde{K}+Z_{i j} \Phi_{i} \bar{\Phi}_{j}+\left(Y_{i j} \Phi_{i} \Phi_{j}+\text { h.c. }\right)+\ldots, \\
& W=\tilde{W}+\tilde{\mu}_{i j} \Phi_{i} \Phi_{j}+\tilde{\lambda}_{i j k} \Phi_{i} \Phi_{j} \Phi_{k}+\ldots
\end{aligned}
$$

Here all coefficients in the expansion are generic functions of $S$ and $T$, and the ellipses stands for the terms of higher order. Although the wavefunction factor $Z_{i j}$ can have an off-diagonal element in general, we assume it is diagonal, viz $Z_{i j}=Z_{i} \delta_{i j}$, for the sake of simplicity. At any rate, off-diagonal elements are required to be small, roughly smaller than $10^{-2} Z_{i}$, to avoid too large flavor changing neutral currents.

The terms $\tilde{W}$ and $\tilde{\mu}_{i j} \Phi_{i} \Phi_{j}$ in the superpotential vanish in perturbation theory. Thus they are purely due to nonperturbative dynamics of the integrated hidden sector. Note that a nontrivial nonperturbative superpotential $\tilde{W}$ of $S$ and $T$ is essential for SUSY breaking by nonzero $F$-components (here the indices $I, J=S, T$ ):

$$
\bar{F}_{I}=e^{\tilde{K} / 2}|\tilde{W}|\left(\bar{\partial}_{I} \partial_{J} \tilde{K}\right)^{-1}\left(\partial_{J} \tilde{K}+\partial_{J} \ln \tilde{W}\right) .
$$

In string theory, $\mathrm{CP}$ corresponds to a discrete gauge symmetry [20, which is an element of higher dimensional Lorentz, general coordinate transformation, and Yang-Mills groups. As a result, there is no $\mathrm{CP}$-violating bare parameter in string theory and $\mathrm{CP}$ must be broken spontaneously. Spontaneous CP violation would occur through the complex vacuum values of superheavy (Kaluza-Klein or stringy) fields and/or through the vacuum values of light fields. If CP violation is entirely due to light fields, one can study CP violation using a CPinvariant effective lagrangian of light fields. However if some of superheavy fields develop CP-violating values, after integrating out them, CP would appear to be explicitly broken in the effective lagrangian of light fields. Here we do not make any assumption on the nature of $\mathrm{CP}$ violation, and thus allow all complex parameters in the Kähler potential and the superpotential to have the phases of order unity in general.

Now one can integrate out $S$ and $T$ to obtain the effective lagrangian of observable fields, including soft SUSY breaking terms induced by nonzero values of the auxiliary components $F_{S, T}$. The resulting effective superpotential $W_{\text {eff }}$ (see eq. (1)) is given by [21, 22]: 


$$
\begin{aligned}
\lambda_{i j k} & =e^{-i \xi} e^{\tilde{K} / 2} \tilde{\lambda}_{i j k}, \\
\mu_{i j} & =\mu_{1 i j}+\mu_{2 i j}+\mu_{3 i j},
\end{aligned}
$$

where $\xi=\arg (\tilde{W})$ and

$$
\begin{aligned}
\mu_{1 i j} & =\lambda_{N i j}\langle N\rangle, \\
\mu_{2 i j} & =\left(m_{3 / 2}-\bar{F}_{I} \bar{\partial}_{I}\right) Y_{i j}, \\
\mu_{3 i j} & =e^{-i \xi} e^{\tilde{K} / 2} \tilde{\mu}_{i j},
\end{aligned}
$$

for the gravitino mass given by $m_{3 / 2}=e^{\tilde{K} / 2}|\tilde{W}|$. Here we consider three possible sources for the bilinear terms in $W_{\text {eff }}$. Note that the $\mu_{1 i j}$-piece is obtained by replacing the singlet field $N$ (if it exists) which has the trilinear coupling $\lambda_{N i j} N \Phi_{i} \Phi_{j}$ by its vacuum value.

For the soft terms of eq. (2), one finds 21, 22

$$
\begin{aligned}
A_{i j k} & =\lambda_{i j k} F_{I} \partial_{I}\left[\ln \left(e^{\tilde{K}} \tilde{\lambda}_{i j k} / Z_{i} Z_{j} Z_{k}\right)\right] \\
B_{i j} & =B_{1 i j}+B_{2 i j}+B_{3 i j} \\
m_{a} & =\frac{1}{2} g_{a}^{2} F_{I} \partial_{I} f_{a}
\end{aligned}
$$

where

$$
\begin{aligned}
& B_{1} / \mu_{1}=A_{N i j} / \lambda_{N i j}, \\
& B_{2} / \mu_{2}=F_{I} \partial_{I}\left[\ln \left(e^{\tilde{K} / 2} \mu_{2 i j} / Z_{i} Z_{j}\right)\right]-m_{3 / 2}, \\
& B_{3} / \mu_{3}=F_{I} \partial_{I}\left[\ln \left(e^{\tilde{K}} \tilde{\mu}_{i j} / Z_{i} Z_{j}\right)\right]-m_{3 / 2} .
\end{aligned}
$$

In fact, soft parameters given above correspond to those renormalized at a scale around the Planck scale. Thus it is necessary to include renormalization effects when one applies the above formulae for low energy phenomenology. For the SUSY phases $\phi_{A}=\arg \left(A_{i j k} / \lambda_{i j k}\right)$, $\phi_{B}=\arg \left(B_{i j} / \mu_{i j}\right)$, and $\phi_{C}=\arg \left(m_{a}\right)$, one needs to take into account the renormalization group ( $\mathrm{RG}$ ) mixing with other potentially large CP-violating phases, particularly with the KM phase $\delta_{\mathrm{KM}}$ which is of order unity, that occurs at scales between the Planck scale and the weak scale. It turns out that the $R G$ mixing with $\delta_{\mathrm{KM}}$ gives a negligible correction, 
and thus $\phi_{A, B, C}$ at the weak scale remain to be small enough as long as they are less than $10^{-2}-10^{-3}$ at the Planck scale [23].

For the supergravity action invariant under the PQ symmetries $U(1)_{S}$ and $U(1)_{T}$ of eq. (12), the corresponding Kähler potential can be chosen to be invariant. Then the superpotential should be invariant up to a constant phase and the gauge kinetic functions up to imaginary constants. The coefficient functions $\tilde{K}$ and $Z_{i j}=Z_{i} \delta_{i j}$ of the Kähler potential of eq. (13) are largely dominated by PQ-conserving perturbative contributions. Note that nonperturbative PQ-violating corrections are suppressed by $q \equiv q_{S, T}$ which are presumed to be less than $10^{-2}-10^{-3}$. The same is true for the gauge kinetic functions $f_{a}$. Then up to small corrections of $O(q)$ 24,

$$
\begin{aligned}
\tilde{K} & =\tilde{K}\left(S+S^{*}, T+T^{*}\right), \\
Z_{i} & =Z_{i}\left(S+S^{*}, T+T^{*}\right), \\
f_{a} & =k_{a} S+l_{a} T,
\end{aligned}
$$

where $k_{a}$ and $l_{a}$ are some real constants. It is also quite likely that the Yukawa couplings $\tilde{\lambda}_{i j k}$ which are relevant for the SUSY phase $\phi_{A}=\arg \left(A_{i j k} / \lambda_{i j k}\right)$ (see eq. (17)) are also dominated by perturbative contributions, implying

$$
\tilde{\lambda}_{i j k}=\left(h_{i j k}+O(q)\right) \exp \left(a_{i j k} S+b_{i j k} T\right),
$$

where $h_{i j k}$ is a complex constant while the constants $a_{i j k}$ and $b_{i j k}$ are real.

We already noted that $\tilde{W}$ is induced by nonperturbative hidden sector dynamics which would be described by the hidden gauge kinetic functions and the hidden Yukawa couplings whose forms are restricted by $U(1)_{S}$ and $U(1)_{T}$ as those of eqs. (18) and (19). Then the arguments based on $R$-symmetries and dimensional analysis [25] imply that $\tilde{W}$ can be written as

$$
\tilde{W}=\sum_{n=1}^{N_{W}} W_{n}=\sum(1+O(q)) d_{n} e^{\left(k_{n} S+l_{n} T\right)},
$$

where $k_{n}$ and $l_{n}$ are some real constants and $d_{n}$ is a complex constant. 
Since the corrections suppressed by a factor less than $10^{-2}-10^{-3}$ are essentially ignored in our approximation, $\tilde{W}$ includes only the terms such that $\left|W_{n} / W_{1}\right| \geq 10^{-2}-10^{-3}$ where $W_{1}$ denotes the term with the largest vacuum value. The number of such terms, viz $N_{W}$, would depend on some details of hidden sector dynamics.

Let us briefly discuss $N_{W}$ for several simple cases. If the hidden sector contains a simple gauge group $\mathcal{G}_{1}$ whose dynamical mass scale $\Lambda_{1}$ is far above those of other groups, then $\tilde{W} \simeq W_{1} \sim \Lambda_{1}^{3}$ where $W_{1}$ contains the gaugino condensation together with possible matter condensations. In the case that there exists another simple group $\mathcal{G}_{2}$ with $\Lambda_{2}$ comparable to $\Lambda_{1}, \tilde{W}$ contains at least two terms $W_{1,2} \sim \Lambda_{1,2}^{3}$. If the gaugino condensations are largely dominate over other possible contributions, one simply has $N_{W}=2$ associated with the two gaugino condensations of $\mathcal{G}_{1}$ and $\mathcal{G}_{2}$. Even in the case that matter condensations become important, if the fields that transform nontrivially under $\mathcal{G}_{1}$ communicate weakly with those of $\mathcal{G}_{2}$, e.g. communicate only via nonrenormalizable interactions, one still has $N_{W}=2$ but now $W_{1}$ and $W_{2}$ contain both the gaugino and matter condensations of the $\mathcal{G}_{1}$-sector and the $\mathcal{G}_{2}$-sector respectively. As is well known, the simplest case of $N_{W}=1$ suffers from the runaway of the dilaton. Because of this, SUSY breaking in string theories has been discussed mainly in the context of nonperturbative superpotentials with $N_{W}=2$ [7], 8].

\section{SMALL SUSY PHASES IN STRING-INSPIRED SUPERGRAVITY}

We are now ready to discuss the mechanism for suppressing SUSY phases in stringinspired supergravity models. The coefficients of soft terms of eq. (17) show that there are a number of potentially complex quantities which can contribute to the phases

$$
\begin{aligned}
& \phi_{A}=\left\{\arg \left(A_{i j k} / \lambda_{i j k}\right)\right\}, \\
& \phi_{B}=\left\{\arg \left(B_{i j} / \mu_{i j}\right)\right\}, \\
& \phi_{C}=\left\{\arg \left(m_{a}\right)\right\} .
\end{aligned}
$$


First of all, the SUSY breaking order parameters $F_{I}$ can be complex in general. Furthermore, although $\tilde{K}$ and $Z_{i}$ are real functions, their derivatives $\partial_{I} \tilde{K}, \partial_{I} \bar{\partial}_{J} \tilde{K}$, and $\partial_{I} Z_{i}$ can be complex. Besides these, we can have complex $\partial_{I} f_{a}, \partial_{I} \ln \left(\tilde{\lambda}_{i j k}\right)$, and several others. It is then convenient to classify all the relevant (potentially) complex quantities as follows:

$$
\begin{aligned}
& X_{1}: \partial_{I} \tilde{K}, \partial_{I} \bar{\partial}_{J} \tilde{K}, \partial_{I} Z_{i}, \partial_{I} f_{a}, \partial_{I} \ln \left(\tilde{\lambda}_{i j k}\right) \\
& X_{2}: \partial_{I} \ln (\tilde{W}) ; \\
& X_{3}: \partial_{I} \ln \left(\tilde{\mu}_{i j}\right) ; \\
& X_{4}: \partial_{I} \ln \left(Y_{i j}\right), \bar{\partial}_{I} \ln \left(Y_{i j}\right), \partial_{I} \bar{\partial}_{J} \ln \left(Y_{i j}\right)
\end{aligned}
$$

If $X_{1}$ and $X_{2}$ are all real, then $\phi_{A}$ and $\phi_{C}$ would vanish. The phase $\phi_{B}$ is affected also by $X_{3}$ and $X_{4}$, and thus making it small requires more conditions.

Let us consider $X_{1}$ first. It is rather easy to see that all quantities in $X_{1}$ are real up to corrections of $O(q)$ for $\tilde{K}, Z_{i}, f_{a}$, and $\tilde{\lambda}_{i j k}$ restricted by $U(1)_{S, T}$ as eqs. (18) and (19).

Let us consider $X_{2}=\partial_{I} \ln (\tilde{W})$. For $\tilde{W}=\sum_{1}^{N_{W}} W_{n}$, one has

$$
X_{2}=\left(\sum W_{n} \partial_{I} \ln \left(W_{n}\right)\right) /\left(\sum W_{n}\right)
$$

For $W_{n}$ of eq. (20), $\partial_{I} \ln \left(W_{n}\right)$ is real up to corrections of $O(q)$. Then $X_{2}$ would be real up to corrections of $O(q)$ if the relative phases $\arg \left(W_{n} / W_{m}\right)$ are CP-conserving.

Interestingly enough, for a relatively small value of $N_{W}$ which would be the most interesting case in view of its simplicity, CP-conserving relative phases can be achieved dynamically. To see this, let us consider the case that $\tilde{W}=W_{1}+W_{2}$. In this case of $N_{W}=2$, the standard scalar potential in supergravity gives the following potential of the model-independent axion $\operatorname{Im}(S)$ :

$$
V_{\text {axion }}=\Omega\left[\cos \left(\arg \left(W_{2} / W_{1}\right)\right)+O(q)\right]
$$

where $\arg \left(W_{2} / W_{1}\right)=\left(k_{2}-k_{1}\right) \operatorname{Im}(S)+\delta$, and $\Omega$ and $\delta$ are real functions which are independent of $\operatorname{Im}(S)$. (See eq. (20) for the notations.) Clearly minimizing this axion potential leads to a CP-conserving value of $\arg \left(W_{2} / W_{1}\right)$ up to $O(q)$, and thus a real $X_{2}$. Note that here 
$\delta=\arg \left(d_{2} / d_{1}\right)+\left(l_{2}-l_{1}\right) \operatorname{Im}(T)$ is of order unity in general, but it is dynamically relaxed to a $\mathrm{CP}$ conserving value by the vacuum value of $\operatorname{Im}(S)$. This is quite similar to the Peccei-Quinn mechanism [26] in the axion solution to the strong CP problem, in which $\bar{\theta}$ is dynamically relaxed to zero by the axion vacuum value.

In the above, we have shown that $X_{1}$ and $X_{2}$ are real up to corrections of $O(q)$, and thus $\phi_{A, C}=O(q)$, if SUSY is broken by the auxiliary components of $S$ and $T$ which are induced by a nonperturbative superpotential with $N_{W}=2$. The discussion of the remained phase $\phi_{B}$ is more model-dependent since there are a variety of ways [27] to generate the bilinear terms $\mu_{i j} \Phi_{i} \Phi_{j}$ in the effective superpotential of eq. (1). In the minimal supersymmetric standard model, the only allowed bilinear term is the so-called $\mu$-term, $\mu H_{1} H_{2}$, of the two Higgs superfields $H_{1}$ and $H_{2}$. In the previous section, we have noted three sources for $\mu$, viz $\mu_{1,2,3}$. (See eqs. (15) and (16). Here and in the following, we assume that $\mu H_{1} H_{2}\left(B h_{1} h_{2}\right)$ is the only bilinear term in $W_{\text {eff }}\left(\mathcal{L}_{\text {soft }}\right)$, and thus omit $i j$ indices of the coefficients $\mu_{i j}$ and $B_{i j}$.) If all of $\mu_{1,2,3}$ give important contributions to $\mu$, there would not be any reason for $\phi_{B}$ small. Thus here we consider three simple scenarios in which one of $\mu_{1,2,3}$ dominates over the other two by a factor larger than $10^{2}$ to $10^{3}$.

In the first case that $\mu_{1}=\lambda_{N}\langle N\rangle$ dominates, $\phi_{B}$ simply corresponds to $\phi_{A}$ and thus is of $O(q)$.

In the second case that $\mu_{2}=\left(m_{3 / 2}-\bar{F}_{I} \bar{\partial}_{I}\right) Y$ dominates, $\phi_{B}$ would receive additional contribution from $X_{4}$. Orbifold compactifications give $Y=0$ [21] and thus they do not correspond to this case. For Calabi-Yao compactifications, it has been pointed out that in $(2,2)$ models $Y$ is related to some Yukawa couplings [21] (by world sheet Ward identities) which are constants up to corrections of $O(q)$. This leads to $X_{4}=O(q)$ [22] and thus $\phi_{B}=O(q)$. Thus in the case of $\mu_{2}$-domination, $\phi_{B}$ can be small at least in $(2,2)$ Calabi-Yao compactification models

In the third case that $\mu_{3}$ dominates, $\phi_{B}$ would receive a contribution from $X_{3}=\partial_{I} \ln (\tilde{\mu})$. Since $\tilde{\mu}$ is due to nonperturbative hidden sector dynamics, using the same arguments applied 
for the nonperturbative superpotential $\tilde{W}$, it can be written as

$$
\tilde{\mu}=\sum \tilde{\mu}_{n}
$$

where $\tilde{\mu}_{n}=(1+O(q)) z_{n} \exp \left(x_{n} S+y_{n} T\right)$. Here $x_{n}$ and $y_{n}$ are some real constants while $z_{n}$ is a complex constant. For hidden sector yielding $\tilde{W}$ with $N_{W}=2$, which is the most interesting case for us, it is expected that $\tilde{\mu}$ also has two terms, $\tilde{\mu}=\tilde{\mu}_{1}+\tilde{\mu}_{2}$. Again $\partial_{I} \ln \left(\tilde{\mu}_{1,2}\right)$ are real up to $O(q)$. However to have a real $X_{3}=\partial_{I} \ln (\tilde{\mu})$, one needs the relative phase $\arg \left(\tilde{\mu}_{1} / \tilde{\mu}_{2}\right)$ to be CP conserving. For $\tilde{W}=W_{1}+W_{2}$, the relative phase $\arg \left(W_{1} / W_{2}\right)$ could be relaxed to a $\mathrm{CP}$ conserving value by the vacuum value of $\operatorname{Im}(S)$. For $\tilde{\mu}$, we do not have any such mechanism, implying $\phi_{B}$ would not be small enough unless further assumptions are made.

The above discussion of $\phi_{B}$ implies that perhaps the first case of $\mu_{1}$-domination is most attractive for the purpose of small $\phi_{B}$, while the third case of $\mu_{3}$-domination is least attractive.

So far, our discussion has been restricted to the case that SUSY is broken by the auxiliary components of $S$ and $T$ induced by a nonperturbative superpotential $\tilde{W}$ with $N_{W}=2$. In fact, the discussion can be easily generalized to more general cases with additional moduli and/or a larger $N_{W}$.

Let us suppose an additional modulus $M$ and define the corresponding PQ symmetry $U(1)_{M}: M \rightarrow M+i \alpha_{M}$. This additional modulus can affect our previous analysis by two ways: (a) it can directly affect SUSY phases by having a nonzero auxiliary component $F_{M}$, (b) it can affect $F_{I}(I=S, T)$ via the wave function mixing with $S$ and $T$. Let $q_{M}$ denote a factor representing the size of $U(1)_{M}$-breaking. Then including $M$ in the analysis, it is easy to see that SUSY phases receive additional contributions which are of the order of either $q_{M} F_{M} / m_{3 / 2}$ or $q_{M} \bar{\partial}_{I} \partial_{M} \tilde{K} / \bar{\partial}_{I} \partial_{I} \tilde{K}$.

What would be the typical size of the PQ symmetry breaking factor $q_{M}$ ? For a Kähler class modulus $M_{K}$ that is associated with the deformation of the Kähler class of the internal space, the pseudoscalar component $\operatorname{Im}\left(M_{K}\right)$ comes from the zero modes of the antisymmetric 
tensor field. Then the corresponding PQ symmetry is broken by world sheet instantons [5], leading to $q_{M_{K}}=e^{-2 \pi M_{K}}$ which can be small enough, say less than $10^{-2}-10^{-3}$, for a moderately large value of $\operatorname{Re}\left(M_{K}\right)$. For another type of moduli, the complex structure moduli $M_{C}$ that is associated with the deformation of the complex structure, the size of $q_{M_{C}}$ is somewhat model-dependent. For orbifold compactifications, $q_{M_{C}}$ is still exponentially small due to the modular symmetry $S L(2, Z)$ [28]. However for Calabi-Yao cases, $q_{M_{C}}$ can be of order unity even at leading order approximation [5]. As a result, to achieve small SUSY phases in Calabi-Yao compactification, one needs to assume that the complex structure moduli give negligible contribution to SUSY breaking, viz $F_{M_{C}} / m_{3 / 2} \leq 10^{-2}-10^{-3}$, and also have small wave function mixing with the Kähler moduli $M_{K}$, viz $\bar{\partial}_{M_{C}} \partial_{M_{K}} \tilde{K} / \bar{\partial}_{M_{K}} \partial_{M_{K}} \tilde{K} \leq$ $10^{-2}-10^{-3}$.

Finally let us consider the dynamical relaxation of the relative phases $\arg \left(W_{n} / W_{n}\right)$ for $N_{W}>2$. Suppose we have $N_{A}$ axion-like fields $\vec{A}=\left(A_{1}, \ldots, A_{N_{A}}\right)$, being the pseudoscalar components of $S, T$ and other possible moduli. If the PQ symmetries, $U(1)_{\vec{A}}: \vec{A} \rightarrow \vec{A}+$ $\vec{\alpha}$, are good approximate symmetries as $U(1)_{S, T}$ are, then the axion-dependence of the nonperturbative superpotential $\tilde{W}=\sum_{1}^{N_{W}} W_{n}$ would be given by $W_{n} \sim e^{i \vec{c}_{n} \cdot \vec{A}}$ where $\vec{c}_{n}$ is a real constant vector. Let $N$ denote the number of linearly independent vectors among $\left\{\vec{c}_{n}-\vec{c}_{m}\right\}$. Obviously $1 \leq N \leq N_{A}$. Then analyzing the potential of $\vec{A}$ as we did in the case of $N_{W}=2$, one can see that if

$$
N_{W} \leq N+1
$$

all the relative phases $\arg \left(W_{n} / W_{m}\right)$ are relaxed to $\mathrm{CP}$ conserving values by the vacuum values of $\vec{A}$.

\section{CONCLUSION}

In summary, we have discussed a mechanism for suppressing SUSY phases in stringinspired supergravity models in which supersymmetry is broken by the auxiliary components of the dilaton and/or moduli superfields. The key ingredients of the mechanism are: 
(i) the approximate PQ symmetries nonlinearly realized for the dilaton and moduli superfields, (ii) dynamical relaxation of the relative phases $\arg \left(W_{n} / W_{m}\right)$ for the nonperturbative superpotential $\tilde{W}=\sum_{1}^{N_{W}} W_{n}$ of the dilaton and moduli superfields.

If supersymmetry breaking is dominated by the dilaton and/or some of the Kähler class moduli whose nonperturbative superpotential has $N_{W}=2$, which would be perhaps the most interesting case in view of its simplicity (in fact, many of the SUSY breaking scenarios which have been discussed in the context of string theory belong to this category [7, \&]), the SUSY phases $\phi_{A}$ and $\phi_{C}$ are exponentially suppressed for moderately large values of the moduli. A similar suppression can occur for the remained phase $\phi_{B}$ depending upon how the $\mu$-term is generated. One would then have SUSY phases less than $10^{-2}-10^{-3}$ in a quite natural manner. An interesting feature of this mechanism is that it is completely independent of how CP is broken [29]. As we have noted, our mechanism for suppressing SUSY phases can be implemented in more general cases with $N_{W}>2$ if $N_{W} \leq N_{A}+1$ for $N_{A}$ denoting the number of available axion-like fields.

\section{ACKNOWLEDGMENTS}

This work is supported in part by KOSEF through CTP at Seoul National University. 


\section{REFERENCES}

[1] For reviews, see J. E. Kim, Phys. Rep. 150 (1987) 1; H. Y. Cheng, Phys. Rep. 158 (1988) 1; R. D. Peccei, in: CP violation, ed. C. Jarlskog (World Scientific, Singapore, 1989).

[2] E. Witten, Nucl. Phys. B188 (1981) 513.

[3] For a recent discussion of the SUSY phase problem, see for instance R. Garisto, TRIPP-93-24 (1993).

[4] H. P. Nilles, Phys. Rep. 110 (1984) 1.

[5] M. B. Green, J. H. Schwarz, and E. Witten, Superstring Theory (Cambridge University Press, 1987).

[6] K. Choi, SNUTP 93-85 (1993).

[7] J. Louis, in Proc. 1991 DPF Meeting (World Scientific, 1992) and references there in.

[8] B. de Carlos, J. A. Casas, and C. Muñoz, Nucl. Phys. B399 (1993) 623.

[9] M. Dugan, B. Grinstein and L. Hall, Nucl. Phys. B255 (1985) 413.

[10] W. Buchmuller and D. Wyler, Phys. Lett. B121 (1983) 321; J. Polchinski and M. B. Wise, Phys. Lett. B125 (1983) 393; F. del Aguila et. al., Phys. Lett. B126 (1983) 71.

[11] S. Weinberg, Phys. Rev. Lett. 63 (1989) 2333.

[12] R. Arnowitt, J. L. Lopez and D. V. Nanopoulos, Phys. Rev. D42 (1990) 2423; R. Arnowitt, M. J. Duff and K. S. Stelle, Phys. Rev. D43 (1991) 3085.

[13] R. L. Jaffe and A. Manohar, Nucl. Phys. B337 (1990) 509 and references there in.

[14] The strange quark contribution to the neutron EDM in multi-Higgs doublet models has been considered by X. He, B. H. J. McKellar and S. Pakvasa, Phys. Lett. B254 (1991) 231.

[15] Y. Kizukuri and N. Oshimo, Phys. Rev. D46 (1992) 3025; Phys. Rev. D45 (1992) 1806. 
[16] M. Dine, N. Seiberg, X.-G. Wen, and E. Witten, Nucl. Phys. B278 (1986) 769; Nucl. Phys. B289 (1987) 319.

[17] E. Witten, Phys. Lett. B149 (1984) 351.

[18] K. Choi and J. E. Kim, Phys. Lett. B154 (1985) 393; Phys. Lett. B165 (1985) 71.

[19] A. Font et. al., Phys. Lett. B245 (1990) 401; S. Ferrara et. al., Phys. Lett. B245 (1990) 409.

[20] K. Choi, D. B. Kaplan, and A. E. Nelson, Nucl. Phys. B391 (1993) 515; M. Dine, R. G. Leigh, and D. A. MacIntire, Phys. Rev. Lett. 69 (1992) 2030.

[21] V. Kaplunovsky and J. Louis, Phys. Lett. B306 (1993) 269.

[22] A. Brignole, L. E. Ibáñez, and C. Muñoz, FTUAM-26/93 (1993).

[23] K. Enqvist, D. V. Nanopoulos, and A. Raychaudhuri, Phys. Lett. B151B (1985) 210; S. Bertolini and F. Vissani, SISSA preprint 173/93/EP (1993).

[24] C. P. Burgess, A. Font, and F. Quevedo, Nucl. Phys. B272 (1986) 661; H. P. Nilles, Phys. Lett. B180 (1986) 240.

[25] For a review, see for example, D. Amati et. al., Phys. Rep. 162 (1988) 169.

[26] R. D. Peccei and H. R. Quinn, Phys. Rev. lett. 38 (1977) 1440.

[27] J. E. Kim and H. P. Nilles, Phys. Lett. B138 (1984) 150; G. F. Giudice and A. Masiero, Phys. Lett. B206 (1988) 480; E. J. Chun, J. E. Kim, and H. P. Nilles, Nucl. Phys. B370 (1992) 105 .

[28] L. E. Ibáñez and D. Lüst, Nucl. Phys. B382 (1992) 305.

[29] For a solution to the small SUSY phase problem utilizing spontaneously broken CP, see K. S. Babu and S. Barr, BA-93-42 (1993). 